\title{
Cystic fluid neutrophil gelatinase-associated lipocalin (NGAL) concentration in differential diagnosis of pancreatic cystic lesions: a new factor enters the scene?
}

\author{
Michal Lipinski ${ }^{1}$, Malgorzata Degowska ${ }^{1}$, Grazyna Rydzewska ${ }^{1,2}$ \\ ${ }^{1}$ Department of Gastroenterology, Central Clinical Hospital of the Ministry of Interior and Administration, Warsaw, Poland \\ ${ }^{2}$ The Faculty of Medicine and Health Sciences, Jan Kochanowski University, Kielce, Poland
}

Gastroenterology Rev 2018; 13 (2): 132-136

DOI: https://doi.org/10.5114/pg.2017.68805

Key words: cystic lesions, pancreas, endoscopic ultrasound, neutrophil gelatinase-associated lipocalin, endosonography.

\begin{abstract}
Address for correspondence: Michal Lipinski MD, PhD, Department of Gastroenterology, Central Clinical Hospital of The Ministry of Interior and Administration, 137 Woloska St, 02-507 Warsaw, Poland, phone: +48 2250812 40, fax: +48 2250810 40,
\end{abstract} e-mail: michal7lipinski@yahoo.com

\begin{abstract}
Introduction: Neutrophil gelatinase-associated lipocalin - $25 \mathrm{kDa}$ peptide - is at present one of the most fascinating and unrecognised proteins implicated in the process of tumour development. Precise assessment of pancreatic cystic lesions is crucial for selecting available treatment options, such as conservative therapy or surgical resection.

Aim: To determine the utility of NGAL concentration in cyst fluid obtained by endoscopic ultrasound (EUS) with EUS-guided fine-needle aspiration (EUS-FNA) to distinguish neoplastic pancreatic cysts from pseudocysts.

Material and methods: Twenty-two patients underwent EUS and FNA of a pancreatic cystic lesion; 9 of these patients underwent surgical resection, providing a histologic diagnosis of the cystic lesion. Furthermore, the final diagnosis was based on cyst fluid cytology, cyst fluid tumour markers (CEA, CA 72-4, CA 19-9), and medical history. Patients were divided in two groups: cystic neoplasms and inflammatory cysts (pseudocysts).

Results: The final diagnosis was pseudocyst in 7 patients, serous cystadenoma in 4, mucinous cystadenoma in 3, intraductal papillary mucinous neoplasms in 6 patients, and cystic form of pancreatic adenocarcinoma in 2. Cyst fluid analysis of these patients showed that median cyst fluid NGAL for the cystic neoplasm group $(211 \mathrm{ng} / \mathrm{ml} ; n=15)$ was significantly lower $(p=0.02)$ than the inflammatory cystic group $(4689 \mathrm{ng} / \mathrm{ml} ; n=7)$. Correlation analysis showed that only fluid CA $72-4$ was positively related to NGAL $(r=0.8, p<0.01)$.

Conclusions: In this single-centre study, pancreatic cyst fluid NGAL concentration appeared to be useful in distinguishing neoplastic pancreatic cysts from pseudocysts. Larger studies are recommended to evaluate this role further.
\end{abstract}

\section{Introduction}

Pancreatic cystic lesions are usually found during routine abdominal ultrasound or cross-sectional imaging studies. The differential diagnosis of pancreatic cysts includes a very broad range of conditions, i.a. cystic neoplasms, solid neoplasms with cystic degeneration, or non-neoplastic cysts. There is no gold standard test for pancreatic cystic lesions. However, imaging findings, clinical history, and cystic fluid cytopathological and biochemical analyses may be of help in differential diagnosis [1].

Endoscopic ultrasound (EUS) is a source of valuable information about pancreatic cysts [2]. Endoscopic ultrasound-guided fine-needle aspiration (EUS-FNA) can be used to collect pancreatic cystic fluid for cytopathological and biochemical analyses. In some cases, cystic fluid amylase and carcinoembryonic antigen (CEA) levels may also be useful for differential diagnosis [3]. However, there are no reliable biochemical factors or ultrasound criteria that would enable preoperative differentiation of different types of pancreatic cysts. Specific and sensitive cystic fluid markers for the differential diagnosis of pancreatic cysts are still needed.

Neutrophil gelatinase-associated lipocalin (NGAL), a $25-k D a$ peptide, is one of the most fascinating but unrecognised proteins involved in the process of tumour development. Its expression is dysregulated in many benign and malignant diseases. It is now under 
investigation as a diagnostic and prognostic biomarker in many diseases, including inflammatory conditions and cancer, due to its small size, relative stability, and secretory characteristics.

Until now, NGAL has been mainly studied as an inflammatory factor and a marker of kidney damage [4]. However, according to recent studies NGAL plays a potential role in cancer development. It may also exhibit pro-oncogenic or anti-oncogenic actions [5]. In fact, it is the NGAL/MMP-9 (matrix metalloproteinase) complex that is associated with the oncogenic effect [6], while the anti-neoplastic effect of NGAL is related to the inhibition of a pro-neoplastic factor hypoxia-inducible factor 1a (HIF-1a), HIF-1a-dependent vascular endothelial growth factor (VEGF), and focal adhesion kinase (FAK) $[7,8]$.

We hypothesised that NGAL concentration in cystic fluid is a potential diagnostic marker for a broad spectrum of pancreatic cystic lesions. However, there are no studies in the literature evaluating cystic fluid NGAL levels in pancreatic cysts.

\section{Aim}

The purpose of the study was to determine the utility of NGAL concentration measurements in cystic fluid obtained by EUS-FNA in distinguishing between neoplastic pancreatic cysts and pseudocysts.

\section{Material and methods}

We evaluated patients who had been admitted to the pancreatic outpatient clinic and diagnosed with cystic pancreatic lesions between August 2011 and September 2012. Patients were included in the study when EUS-FNA was indicated. We excluded patients who had not consented to the study or patients with too little cystic fluid obtained for analysis. We gathered the following information: EUS characteristics of the cystic lesions, location, and number of lesions. The EUS-FNA was performed with curvilinear echo-endoscopes. Standard 19- or 22-gauge needles were used. We noted the characteristics of the aspirated cystic fluid: volume, colour, and viscosity. Cystic fluids collected with EUS-FNA were sent for cytopathological, biochemical, and NGAL analyses. All patients had estimated glomerular filtration rate (eGFR) within the reference range.

Twenty-two patients underwent EUS-FNA of a pancreatic cystic lesion: nine of those patients underwent surgical resection with subsequent histological examination of the cystic lesion. Furthermore, the final diagnosis was based on cystic fluid cytology, cystic fluid tumour markers (CEA, CA 72-4, CA 19-9), and medical history. Patients were divided into two groups: cystic neoplasms and inflammatory cysts (pseudocysts).
The value of cystic fluid NGAL was correlated to corresponding cytological examination, surgical histopathology (when available), and levels of tumour markers in the cystic fluid. Cystic fluid CEA, Ca19-9, CA 72-4, and amylase levels were measured with an automated analyser in the reference clinical biochemistry laboratory. Some of the cystic fluid was stored before NGAL analysis.

The NGAL assays were performed using a standardised clinical platform (ARCHITECT analyser). The ARCHITECT $^{\circledast}$ assay was characterised by functional sensitivity $<2 \mathrm{ng} / \mathrm{ml}$ ( $20 \%$ coefficient of variation (CV), 95\% confidence).

\section{Statistical analysis}

Student's $t$-test was used to compare average values across groups with $p$-value of 0.05 determining significance. The Spearman correlation coefficient was used to evaluate the relationship between NGAL, tumour markers and amylase. In all analyses, the level of significance was set at $\alpha=0.05$.

\section{Results}

A pseudocyst was diagnosed in 7 patients, serous cystadenoma in 4, mucinous cystadenoma in 3, intraductal papillary mucinous neoplasms in 6 patients, and cystic forms of pancreatic adenocarcinoma were identified in two individuals (Table I).

Cystic fluid analysis of these patients showed that median cystic fluid NGAL for the cystic neoplasm group (211 ng/ml; $n=15)$ was significantly lower $(p=0.02)$ than in the inflammatory cyst group $(4689 \mathrm{ng} / \mathrm{ml}$; $n=7$ ) (Figure 1).

Correlation analysis showed that only fluid CA 72-4 was positively associated with NGAL $(r=0.8, p<0.01)$. Other correlations were as follows: CEA $r=0.006$, CA 19-9 $r=-0.01$, and amylase $r=-0.11$ (Table II).

\section{Discussion}

Increased detection of cystic lesions of the pancreas has led to a rise in the number of pancreatic surgical resections [9]. However, since many cystic lesions are known to be benign, resection may be unjustified in many cases. Thus, it is of the utmost importance to characterise cystic neoplasms and to distinguish cystic neoplasms of the pancreas from pseudocysts.

The NGAL has been suggested as a protein playing an important role in carcinogenesis of various neoplasms and a key player in the development of different cancer types $[10,11]$. In the main part of the analysed dataset NGAL transcripts were significantly higher in most solid tumours compared to the corre- 
Table I. Clinical and pathological features of pancreatic cystic lesions

\begin{tabular}{|c|c|c|c|c|c|}
\hline Parameter & Pseudocysts & Serous cystadenoma & $\begin{array}{c}\text { Mucinous } \\
\text { cystadenoma }\end{array}$ & $\begin{array}{l}\text { Intraductal papillary } \\
\text { mucinous neoplasms }\end{array}$ & $\begin{array}{c}\text { Cystic form } \\
\text { of pancreatic } \\
\text { adenocarcinoma }\end{array}$ \\
\hline$N$ & 7 & 4 & 3 & 6 & 2 \\
\hline $\begin{array}{l}\text { Age, mean } \\
\text { [years] }\end{array}$ & 54 & 56 & 60.6 & 75 & 70 \\
\hline Sex & $\begin{array}{l}\text { Women: } 2 \\
\text { Men: } 5\end{array}$ & $\begin{array}{l}\text { Women: } 4 \\
\text { Men: } 0\end{array}$ & $\begin{array}{l}\text { Women: } 1 \\
\text { Men: } 2\end{array}$ & $\begin{array}{l}\text { Women: } 6 \\
\text { Men: } 0\end{array}$ & $\begin{array}{l}\text { Women: } 1 \\
\text { Men: } 1\end{array}$ \\
\hline Site & $\begin{array}{c}\text { Head: } 2 \\
\text { Body: } 1 \\
\text { Body/tail: } 2 \\
\text { Tail: } 1\end{array}$ & $\begin{array}{c}\text { Head: } 1 \\
\text { Head/body: } 1 \\
\text { Body: } 2\end{array}$ & $\begin{array}{c}\text { Body: } 1 \\
\text { Body/tail: } 1 \\
\text { Tail: } 1\end{array}$ & $\begin{array}{c}\text { Head: } 1 \\
\text { Head/body: } 1 \\
\text { Body: } 1 \\
\text { Body/tail: } 1 \\
\text { Tail: } 2\end{array}$ & Head: 2 \\
\hline $\begin{array}{l}\text { Size }(a \times b) \\
{\left[\mathrm{mm}^{2}\right]}\end{array}$ & 2112 & 1005 & 1222 & 243 & 1022 \\
\hline Signs & $\begin{array}{c}\text { Acute and chronic } \\
\text { pancreatitis, pain, } \\
\text { weight loss }\end{array}$ & $\begin{array}{l}\text { Pain, acute } \\
\text { pancreatitis }\end{array}$ & $\begin{array}{l}\text { Pain, acute } \\
\text { pancreatitis }\end{array}$ & Pain, weight loss & Anaemia, weight loss \\
\hline
\end{tabular}

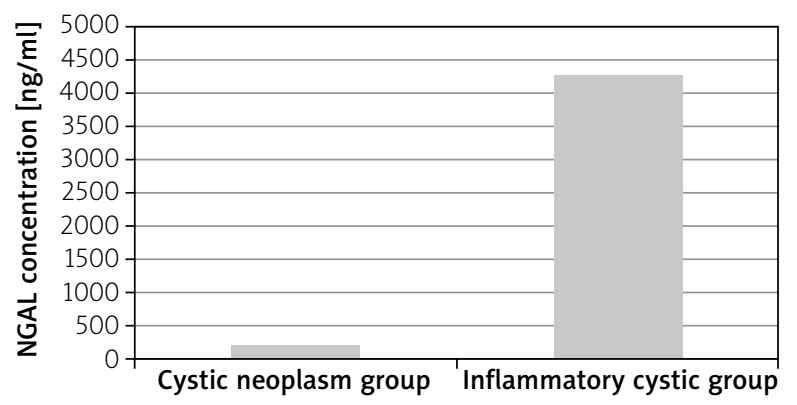

Figure 1. Pancreatic cyst fluid NGAL concentration

sponding normal tissues. Moreover, concordance of NGAL at both mRNA and protein levels was observed in various types of cancer: colorectal, lung, liver, ovarian, and pancreatic [10]. All metastatic tumours showed reduction in NGAL expression compared to matched primary lesions. The NGAL can also be detected in bile and might be useful as a novel biomarker distinguishing between benign and malignant biliary obstruction [12].

The oncogenic effect of NGAL may be related to the NGAL/MMP-9 complex. NGAL's ability to form a dimeric complex with MMP-9 protects MMP-9 from autodegradation and, consequently, leads to increased gelatinolytic action of MMP-9 on the extracellular matrix [13] It has been shown that NGAL may promote cancer development in a variety of different cancer types through this mechanism $[14,15]$. Conversely, it has been demonstrated that the anticancer actions of NGAL are related to its ability to inhibit the pro-neoplastic HIF-1a factor, synthesis of HIF-1a-dependent VEGF, and phosphorylation of FAK kinase [8].
Table II. Correlation coefficient between NGAL and tumour markers

\begin{tabular}{ccccc} 
NGAL & CEA & CA 19-9 & CA 72-4 & Amylase \\
\hline$r$ & -0.006 & -0.01 & $0.8(p<0.01)$ & -0.11
\end{tabular}

The NGAL is also an acute-phase protein [16], it plays a role in inflammation [17], and it becomes upregulated in the lung under inflammatory conditions [18]. Numerous factors ranging from proinflammatory cytokines, such as interleukins, tumour necrosis factor- $\alpha$, and interferons, to vitamins, like retinoic acid, regulate NGAL expression. NGAL is also known as a protective factor in oxidative stress [19].

Numerous factors ranging from proinflammatory cytokines, such as interleukins, tumour necrosis factor- $\alpha$, and interferons, to vitamins, like retinoic acid, regulate NGAL expression.

The NGAL is a sensitive marker of ischaemic damage to renal tubular epithelium and thereby indicates NGAL as an early, next-generation predictor of acute kidney injury $[20,21]$ as well as other diseases, such as acute pancreatitis [22, 23].

Our results show that the concentration of NGAL in cystic fluid was significantly elevated in inflammatory cysts (pseudocysts) compared to cystic neoplasms. This observation may be explained by the fact that NGAL is a proinflammatory molecule and its expression can be induced by several cytokines and growth factors, including interleukin-1 [24], transforming growth factor $\alpha$ (TGF- $\alpha$ ) [25], and tumour necrosis factor $\alpha(T N F-\alpha)[26]$. Results suggest that NGAL may 
be helpful in distinguishing pancreatic cystic lesions and is a candidate marker for identification of pseudocysts.

Our results show that only cystic fluid CA 72-4 correlated positively with NGAL. No other significant correlations involving CA 19-9, CEA, or amylase were observed.

This is the first study investigating cystic NGAL levels in a broad spectrum of pancreatic cystic lesions. However, it has some important limitations, e.g. selection bias because we included only patients who had undergone EUS-FNA. Those patients represent a subset of a population with suspicious or atypical lesions on initial evaluation. Our results may not reflect the entire population of patients with pancreatic cysts. The second limitation concerns the small number of patients in some subsets of the study group because some cystic lesions, including cystic form of pancreatic adenocarcinoma, were very rare. EUS-FNA was performed only in atypical pseudocysts without clear history of pancreatitis or during EUS-guided cystogastrostomy. Finally, although we measured cystic NGAL levels, we did not measure serum NGAL levels for comparison and correlation analyses.

Although NGAL elevation in cystic fluid lowered the suspicion of neoplastic cyst, when a high concentration of NGAL is present, this is admittedly still not sufficiently reassuring in lots of patients. This implies that caution should be used in these cases. Further studies are needed to assess factors that confirm (hypothetically combined with tumour markers such as Ca 72-4) the high risk of neoplastic pancreatic tumours.

\section{Conclusions}

In this single-centre study, NGAL concentration in pancreatic cyst fluid appeared to be useful for distinguishing between neoplastic pancreatic cysts and pseudocysts. Larger studies are recommended to further evaluate this role.

\section{Conflict of interest}

The authors declare no conflicts of interest.

\section{References}

1. Soyer OM, Baran B, Ormeci AC, et al. Role of biochemistry and cytological analysis of cyst fluid for the differential diagnosis of pancreatic cysts: a retrospective cohort study. Medicine 2017; 96: e5513.

2. Kirtane T, Bhutani MS. EUS for pancreatic cystic neoplasms: the roadmap to the future is much more than just a few shades of gray. Asian Pac J Trop Med 2016; 9: 1218-21.

3. Oppong KW, Dawwas MF, Charnley RM, et al. EUS and EUS-FNA diagnosis of suspected pancreatic cystic neoplasms: is the sum of the parts greater than the CEA? Pancreatology 2015; 15: 531-7.

4. Antonucci E, Lippi G, Ticinesi A, et al. Neutrophil gelatinase-associated lipocalin (NGAL): a promising biomarker for the early diagnosis of acute kidney injury (AKI). Acta Biomed 2014; 85: 289-94.

5. Bolignano D, Donato V, Lacquaniti A, et al. Neutrophil gelatinase-associated lipocalin (NGAL) in human neoplasias: a new protein enters the scene. Cancer Lett 2010; 288: 10-6.

6. Yan L, Borregaard N, Kjeldsen L, et al. The high molecular weight urinary matrix metalloproteinase (MMP) activity is a complex of gelatinase B/MMP-9 and neutrophil gelatinase-associated lipocalin (NGAL). Modulation of MMP-9 activity by NGAL. J Biol Chem 2001; 276: 37258-65.

7. Venkatesha S, Hanai J, Seth P, et al. Lipocalin 2 antagonizes the proangiogenic action of ras in transformed cells. Mol Cancer Res 2006; 4: 821-9.

8. Tong Z, Kunnumakkara AB, Wang $\mathrm{H}$, et al. Neutrophil gelatinase-associated lipocalin: a novel suppressor of invasion and angiogenesis in pancreatic cancer. Cancer Res 2008; 68: 6100-8.

9. BalcomJH, Rattner DW, Warshaw AL, et al. Ten-year experience with 733 pancreatic resections: changing indications, older patients, and decreasing length of hospitalization. Arch Surg 2001; 136: 391-8.

10. Candido S, Maestro R, Polesel J, et al. Roles of neutrophil gelatinase-associated lipocalin (NGAL) in human cancer. Oncotarget 2014; 5: 1576-94.

11. Wysocka O, Kulbacka J, Saczko J. Adjuvant, neoadjuvant, and experimental regimens in overcoming pancreatic ductal adenocarcinoma. Gastroenterology Rev 2016; 11: 155-62.

12. Zabron AA, Horneffer-van der Sluis VM, Wadsworth CA, et al. Elevated levels of neutrophil gelatinase-associated lipocalin in bile from patients with malignant pancreatobiliary disease. Am J Gastroenterol 2011; 106: 1711-7.

13. Yan L, Borregaard N, Kjeldsen L, et al. The high molecular weight urinary matrix metalloproteinase (MMP) activity is a complex of gelatinase B/MMP-9 and neutrophil gelatinase-associated lipocalin (NGAL). Modulation of MMP-9 activity by NGAL. J Biol Chem 2001; 276: 37258-65.

14. Kubben FJ, Sier CF, Hawinkels LJ, Tschesche H, et al. Clinical evidence for a protective role of lipocalin-2 against MMP-9 autodegradation and the impact for gastric cancer. Eur J Cancer 2007; 43: 1869-76.

15. Candido S, Maestro R, Polesel J, et al. Roles of neutrophil gelatinase-associated lipocalin (NGAL) in human cancer. Oncotarget 2014; 5: 1576-94.

16. Liu Q, Nilsen-Hamilton M. Identification of a new acute phase protein. J Biol Chem 1995; 270: 22565-70.

17. Cowland JB, Muta T, Borregaard N. IL-1 beta-specific up-regulation of neutrophil gelatinase-associated lipocalin is controlled by IkappaB-zeta. J Immunol 2006; 176: 5559-66.

18. Chan YR, Liu JS, Pociask DA, et al. Lipocalin 2 is required for pulmonary host defense against Klebsiella infection. J Immunol 2009; 182: 4947-56.

19. Roudkenar MH, Halabian R, Ghasemipour Z, et al. Neutrophil gelatinase-associated lipocalin acts as a protective factor against $\mathrm{H}(2) \mathrm{O}(2)$ toxicity. Arch Med Res 2008; 39: 560-6. 
20. Bolignano D, Donato V, Coppolino G, et al. Neutrophil gelatinase-associated lipocalin (NGAL) as a marker of kidney damage. Am J Kidney Dis 2008; 52: 595-605.

21. Soto K, Papoila AL, Coelho S, et al. Plasma NGAL for the diagnosis of AKI in patients admitted from the emergency department setting. Clin J Am Soc Nephrol 2013; 8: 2053-63.

22. Lipinski M, Rydzewska-Rosolowska A, Rydzewski A, et al. Urinary neutrophil gelatinase-associated lipocalin as an early predictor of disease severity and mortality in acute pancreatitis. Pancreas 2015; 44: 448-52.

23. Rosołowski M, Lipiński M, Dobosz $M$, et al. Management of acute pancreatitis (AP) - Polish Pancreatic Club recommendations. Gastroenterology Rev 2016; 11: 65-72.

24. Bando $M$, Hiroshima $Y$, Kataoka $M$, et al. Interleukin-1alpha regulates antimicrobial peptide expression in human keratinocytes. Immunol Cell Biol 2007; 85: 532-7.

25. Sorensen OE, Cowland JB, Theilgaard-Monch K, et al. Wound healing and expression of antimicrobial peptides/polypeptides in human keratinocytes, a consequence of common growth factors. J Immunol 2003; 170: 5583-9.

26. Landro L, Damas JK, Flo TH, et al. Decreased serum lipocalin-2 levels in human immunodeficiency virus-infected patients: increase during highly active anti-retroviral therapy. Clin Exp Immunol 2008; 152: 57-63.

Received: 22.06 .2017

Accepted: 23.06.2017 